\title{
Diagnosing/Recognising High Functioning Autism in Adult Females: Challenging Stereotypes
}

\section{Claire VM Evans-Williams ${ }^{1^{*}}$ and Damien J Williams ${ }^{2}$}

${ }^{1}$ NHS Tayside, Adult Mental Health Services, Stracathro Hospital, Brechin, UK

${ }^{2}$ School of Medicine, University of St Andrews, St Andrews, UK

*Corresponding author: Evans-Williams CVM, NHS Tayside, Adult Mental Health Services, Stracathro Hospital, Brechin, UK, Tel: 00-44-7583812777; E-mail: cevans21@nhs.net

Received date: 02 May 2016; Accepted date: 27 May 2016; Published date: 03 June, 2016

Copyright: ( $) 2016$ Evans-Williams CVM. This is an open-access article distributed under the terms of the Creative Commons Attribution License, which permits unrestricted use, distribution, and reproduction in any medium, provided the original author and source are credited.

\section{Introduction}

The apparent global rise in Autism has led many to describe it as an epidemic and a major public health concern [1-4]. The extraordinary claim of an epidemic has, however, been widely refuted [5]. Most recently DSM-V unified the four DSM-IV diagnostic categories of Autistic Disorder ("previously referred to as "Autism"), Asperger's Disorder, Childhood Disintegrative Disorder, and Pervasive Developmental Disorder Not Otherwise Specified, into the single category of Autistic Spectrum Disorder. This shift in diagnostic criteria will change the landscape of autism further, in terms of its presentation, prevalence, and treatment. However, in order to ensure appropriate diagnosis and treatment/intervention across the spectrum, it is necessary to address some of the mis-understandings and stereotypes that plague diagnostic and research endeavours with regards autism.

\section{The Nexus of Gender, Age and Ability}

Since its original conception in the 1940's autism has been dominated by a focus on males. Indeed, epidemiological data has consistently highlighted a greater prevalence among males than females, with diagnosis four times more common in males [6-8]. This has led to the notion that Autism is a male disorder, popularised in the "extreme male brain" hypothesis of autism underpinned by the systemiser-empathizer dichotomy [9]. However, since the 1980s, the gender bias in autism has been steadily gaining traction [10,11]. Indeed, Attwood [12] contends that "life on the autism spectrum is not easy for girls and women", who often adjust to the classic profile of autism characteristics differently to males, resulting in a disparate presentation of the condition.

Moreover, our "obstinately persisting misunderstanding" of autism originates largely from clinical and epidemiological studies with children [13]. When the term "Autism" is used it most often conjures a mental image of a male aged 4/5 years with the most extreme/ debilitating presentation encompassing limited verbal ability, selfdirected social isolation, and engagement in repetitive structured activity. Indeed, Klin indicates that "Autism" has also been known as childhood autism, infantile autism, and early infantile autism, which demonstrate the inherent bias toward the earlier years of life [14]. Historically, it has been during childhood that autism is often first noticed, and much is written about children with autism; but autism is first and foremost a disorder of development: the clinical picture can evolve (dramatically) over time, with symptoms changing, emerging, and/or disappearing [15]. Autism is, therefore, lifelong but relatively little is known about autism in adulthood, which has only recently come into focus [16].
Furthermore, the common understanding of autism considers the most severe forms of impairment (i.e., the so-called 'low-functioning' individuals); however, the DSM-V derived spectrum of autism necessarily means that a myriad of presentations will arise, and the classification also accounts for the degree of severity: ranging from those experiencing the most severe presentation to those whom are able to lead independent and fulfilling lives [17]. Indeed, the focus on the less severe end of the spectrum, wherein individuals are often referred to as having so-called "high-functioning autism" (hereafter HFA), has often been overlooked. The very nature of this terminology, however, can be considered misleading, as qualitative parameters such as "low" and "high" may infer an inaccurate perception of an individual's strengths and talents, or challenges and difficulties in the context of that person's life.

Thus, when considered together, it is apparent that one, often overlooked group of individuals with ASD, are female adults with HFA. This may be because women with HFA are at greater risk of missed diagnosis, or being mis-diagnosed [18]. Indeed, they are less easily identifiable diagnostically because they are able to adopt a range of strategies to mask and compensate for the challenges they face, thereby "camouflaging" themselves and leaving little observable evidence to those in their company. Indeed, for women with an underlying, undiagnosed HFA who present at mental health services, the primary focus for treatment and intervention can often be placed on what we see as a surface level presentation of, for example, acute Social Anxiety, OCD, Major Depressive Disorder, or Personality Disorder, with the HFA overshadowed and disguised from sight [19]. This has important clinical implications for the efficacy of support for this often overlooked group of individuals: the foundation of successful treatment/intervention is built upon an accurate diagnosis, which necessitates a true understanding of the HFA phenotype in adult females [20].

Based on personal reflections of the lead author, the following section will attempt to challenge a number of stereotypes and misconceptions that may impede the process of diagnosing HFA in adult females. The list is by no means exhaustive, but rather serves to illustrate some of the key considerations.

\section{Stereotypes in the ASD Phenotype: The Case of High- Functioning Autism in Adult Females}

At a simplistic level, the conceptualization of the dyad of behavioural diagnostic criteria for ASD, according to DSM-V, include:

1. Persistent deficits in social communication and social interaction.

2. Restricted, repetitive patterns of behaviour, interests, or activities. 
The following discussion will focus on these broad criteria and challenge the stereotypes of ASD in the context of diagnosing adult females with HFA.

\section{Persistent Deficits in Social Communication and Social Interaction}

The factors associated with social communication and social interaction can include eye contact, affective empathy, and social relationships.

\section{Deficits in non-verbal communicative behaviours used for social interaction}

One of the most common misconceptions is that the presence of direct eye contact should rule out consideration for HFA diagnosis. However, this is a poor diagnostic staple on which to base clinical decision-making in adult females. Notwithstanding, there can be a variety of reasons as to why even a neuro-typical individual may find it challenging to offer direct eye gaze; nervousness upon meeting a new and unfamiliar person (e.g., a clinician), low self-esteem, or social disengagement resulting from depressive symptomatology. That is not to say that all individuals with HFA find direct eye contact a natural and pleasurable endeavour. Women with HFA often describe how eye contact can be incredibly difficult, with associated feelings of discomfort. The level of sensory detail in the eyes of another may interfere with the ability to adequately process a simultaneous delivery of auditory information during conversation. Conceivably then, looking away from the eyes provides more of a 'mental space' to make sense of the influx of inbound sensory, social information.

Moreover, an understanding that the etiquette of social communication in the neuro-typical world requires eye-contact can lead some females with HFA to learn ways in which to navigate their social world. This may include mimicking and repeated practice and refinement based upon observing the role of eye contact among family members, friends, or by watching their favourite soap characters on TV. This compensatory strategy can sometimes be observed as very structured patterns of eye contact through the use of specific time intervals, or only offered during verbal responses, followed by pauses during which listening occurs. Alternatively, a desire to display socially appropriate behaviour may result in intense staring with little break in eye contact. The adult female with HFA may thus appear to be a natural, non-verbal communicator. Indeed, this naturalistic performance may have been the result of a form of personalised graded exposure, gradually increasing tolerances of discomfort in performing this skill over time, and in various contexts. Such conscious dedication to compensatory strategies results in learned behaviour that reflects the neuro-typical innate ability for non-verbal communication in social interaction. Not excluding the HFA female who finds appropriate eye contact a non-issue (with an apparent ability to execute it with relative ease), we cannot rely on eye contact alone in diagnostic decision-making.

\section{Deficits in social-emotional reciprocity}

It is commonly assumed that individuals with HFA are devoid or significantly lacking in affective empathy. Thus, the presence of affective empathy is frequently used as a basis to dismiss the possibility of HFA in women. In fact, many women with HFA experience what has been referred to as "hyper-empathy" or a sensitive "empathic attunement". This can be a powerful, overwhelming experience that typically, though not exclusively, hones in on the negative emotions of others. It is an ability to absorb, for example, the distress experienced by others which can result in a difficulty differentiating one's own emotions, from the other persons'; a sort of autistic emotional transference. Taking on the emotions of others is an exhausting experience for these women, and can often prompt them to avoid social situations that may give rise to this experience.

Moreover, this empathic sensitivity can extend beyond adult interpersonal relations. For example, many women with HFA share a love of nature and animals, and so tapping into this special interest during clinical assessment may reveal expressed worries, fears, and hopes for a much loved pet. Moreover, women with HFA can be attentive, caring, nurturing mothers of children, which is demonstrable evidence from clinical observation that deficits in social-emotional reciprocity are not necessary for diagnosis of HFA in women. Despite this, many standardised screening measures utilise this facet in delineating differential diagnosis; however, if careful consideration is not taken, a possible HFA diagnosis may be missed.

\section{Deficits in developing and maintaining relationships}

Unlike their male counterparts, adult females with HFA may be more socially motivated by the pursuit of friendships, and have a greater interest in social activities involving others. Similar to neurotypicals, women with HFA can have groups of friends and enjoy spending time with them in a social context, engaging in a variety of social activities. They may also have chosen jobs/careers that involve working collaboratively with others (e.g. a shop assistant, teacher, or indeed a psychologist!). Similarly, women with HFA may also have a desire to seek out romantic, intimate relationships with a partner, with many choosing a traditional route of marriage, and children.

Although women with HFA may enjoy socially interactive relationships, in a given context, these exchanges can be highly energy consuming and lead to premature tiredness and even exhaustion. This is because much of this social interaction is achieved through the use of a vast array of masking and compensatory strategies for managing such social situations making them less identifiable. Unless assessment processes take into account these factors, such as the person-specific compensatory strategies, and post-interaction fatigue, then a paradigm of " presence of empathy=impossibility of ASD" may be concluded.

\section{Restricted, Repetitive Patterns of Behaviour, Interests or Activities}

Exemplars of this category include repetitive motor movements and restricted interests and hobbies.

\section{Stereotyped or repetitive speech, motor movements or use of objects}

Not all women with HFA display the classical depiction of hand flapping, body rocking, or other obvious repetitive motor behaviours. Subtleties of this diagnostic feature may, nonetheless include light leg tapping, foot shaking, or pen waving in certain contexts. However, the idiosyncratic manifestation of such behaviours can be moderated by a number of factors, which may not present themselves in the clinical environment. A feeling of contentment and relaxation may trigger hand flapping for some, whilst leg tapping or pen fidgeting may indicate anxiety or distress for others. Some women with HFA report that repetitive motor movements help them to fade-out and reduce 
sensory input from the external world; however, many women with HFA report no history of significant motor movements. Moreover, many women report a form of habitual suppression of such motor behaviours, which may be linked to cultural expectations of females behaving in a "lady-like" manner. Thus, learning that a behavioural expression is socially unacceptable may be a prominent factor influencing a conscious and/or automatic masking of this ASD feature. Put simply, to exclude a diagnosis of HFA in women on the basis of an absence of repetitive motor behaviours creates an increased risk of missed diagnosis.

\section{Highly restricted, fixated interests that are abnormal in intensity and focus}

Unlike the male phenotype, females with HFA may not be motivated by dissecting the active components of an engine, or systematically working through a list of trains to "spot". Rather, they may be interested in popular culture like following a favoured celebrity, show enthusiasm for pet cats, or have a keen focus on pop music. Such examples illustrate that an intense interest need not be related to the stereotypical male interests, and may actually be more congruent with those of neuro-typicals. While women with HFA might certainly reach obsessive heights with their interests, they generally tend not to be focused on stereotypical interests involving inanimate objects or solitary endeavours.

During the course of clinical assessment, if directed questions are pertaining 'special' interests, the individual may not consider their interest(s) to be unique or necessarily different to others they know; their hobby may not be particularly "special" in that sense. What is most important to consider is the level of intensity, the time spent engaged in that interest and the pleasure gained from that interest. Thus, a broader socially-orientated conceptualisation of "special" interests is required, accounting for intensity, frequency, and pleasure in order to successfully assess the presence of restricted and repetitive interest in women with HFA.

\section{Concluding Comments}

Autism could be a considered a stereotyped disorder: The common view in the minds of the public and often represented in clinical and epidemiological enquiry is of young boys with the triad of severe impairment. However, in reality, ASD covers a wide array of presentations/phenotypes that result from the complex interaction of gender, age, and ability. In order to progress the diagnosis and treatment of individuals with ASD, it is necessary to begin exploring the experiences of those in previously overlooked groups [21]. One such group that epitomizes the nexus of marginality is adult, females, with HFA. These individuals can be fully integrated, valued contributors to society, but they have their own challenges that often go unrecognized due to diagnostic criteria and implementation of compensatory strategies. The adult female with HFA is exceptional!
Throughout their developmental journey they have coped with various challenges that living with HFA brings. It is through careful and delineated adaptation and disguise that they have navigated the world thus far: A paradoxical challenge for the diagnostician, but more so for the adult female with HFA.

\section{References}

1. Elsabbagh M, Divan G, Koh YJ, Kim YS, Kauchali S, et al. (2012) Global prevalence of autism and other pervasive developmental disorders. Autism Res 5: 160-179.

2. Rimland B (1995) Is there an autism epidemic? Autism Research Review International 9: 3.

3. Rimland B (2000) The autism epidemic vaccinations and mercury. Journal of Nutritional and Environmental Medicine 10: 261-266.

4. Newschaffer CJ, Curran LK (2003) Autism: an emerging public health problem. Public Health Rep 118: 393-399.

5. Gernsbacher MA, Dawson M, Goldsmith HH (2005) Three Reasons Not to Believe in an Autism Epidemic. Curr Dir Psychol Sci 14: 55-58.

6. Kanner L (1943) Autistic disturbances of affective contact. Nervous Child 2: 217-250.

7. Asperger H (1944) The "autistic psychopaths" in childhood. Archives of Psychiatry and Nervous Diseases 117: 76-136.

8. Whiteley P, Todd L, Carr K, Shattock P (2010) Gender ratios in autism Asperger syndrome and autism spectrum disorder. Autism Insights 2: 17-24.

9. Baron-Cohen S (2002) The extreme male brain theory of autism. Trends in Cognitive Sciences 6: 248-254.

10. Wing L (1981) Sex ratios in early childhood autism and related conditions. Psychiatry Res 5: 129-137.

11. Lai MC, Baron-Cohen S, Buxbaum JD (2015) Understanding autism in the light of sex/gender. Mol Autism 6: 24 .

12. McKibbin K (2015) Life on the autism spectrum: A guide for girls and women. Jessica Kingsley Publishers, London.

13. Frith U (2003) Autism: Explaining the enigma. (2ndedn), Blackwell Publishing, Oxford.

14. Klin A (2006) Autism and Asperger syndrome: An overview. Rev Bras Psiquiatr 28 Suppl 1: S3-11.

15. Hill E, Frith U (2004) Understanding autism: Insights from mind and brain. Autism: Mind and brain. Oxford University Press, Oxford.

16. Narzisi A (2015) Adults with autism spectrum disorders: Research is the future. Autism Open Access 5: 149.

17. Farley MA, McMahon WM, Fombonne E, Jenson WR, Miller J, et al. (2009) Twenty-year outcome for individuals with autism and average or near-average cognitive abilities. Autism Res 2: 109-118.

18. Luciano CC, Keller R, Politi P, Aguglia E, Magnano F, et al. (2014) Misdiagnosis of high function autism spectrum disorders in adults: An Italian case series. Autism Open Access 4: 131.

19. Lai MC, Baron-Cohen S (2015) Identifying the lost generation of adults with autism spectrum conditions. Lancet Psychiatry 2: 1013-1027.

20. Gould J, Ashton-Smith J (2011) Missed diagnosis or misdiagnosis? Girls and women on the autism spectrum. Good Autism Practice 12: 34-41.

21. Adewale V (2015) Black autistic lives matter. Autism Open Access 5: 141. 